Mathematical Modelling And Analysis

Volume 19 Number 1, February 2014, 75-84

http://dx.doi.org/10.3846/13926292.2014.893263

(c) Vilnius Gediminas Technical University, 2014
Publisher: Taylor\&Francis and VGTU

http://www.tandfonline.com/TMMA

Print ISSN: 1392-6292

Online ISSN: 1648-3510

\title{
Simple Models for Wall Effect in Fiber Suspension Flows
}

\section{Ansis Ozolins and Uldis Strautins}

\author{
University of Latvia \\ Zellu Str. 8, LV-1002 Riga, Latvia \\ E-mail(corresp.): ansiso@inbox.lv \\ E-mail: uldis.strautins@lu.lv
}

Received September 15, 2013; revised January 25, 2014; published online February 20, 2014

\begin{abstract}
Jeffery's equation describes the dynamics of a non-inertial ellipsoidal particle immersed in a Stokes liquid and is used in various models of fiber suspension flow. However, it is not valid in close neighbourhood of a rigid wall. Geometrically impossible orientation states with the fiber penetrating the wall can result from this model. This paper proposes a modification of Jeffery's equation in close proximity to a wall so that the geometrical constraints are obeyed by the solution. A class of models differing in the distribution between the translational and rotational part of the response to the contact is derived. The model is upscaled to a Fokker-Planck equation. Another microscale model is proposed where recoiling from the wall upon the collision is permitted. Numerical examples illustrate the dynamics captured by the models.
\end{abstract}

Keywords: fiber suspension, rheology, wall effect.

AMS Subject Classification: $76 \mathrm{~T} 20$.

\section{Introduction}

Understanding the processes involved in fiber suspension flow near a solid wall is far from complete. Experimental and numerical studies have shown that in sufficiently dilute suspensions, Jeffery's model is verified up to the wall gap to fiber length ratio $h / \ell \geq 3.0$. Close to the wall Jeffery's orbits are perturbed, the period increases by about $10 \%$ at the distance $h / \ell=1.25$. Oscillation of the center of the fiber from and towards the wall is observed at $h / \ell=1.0$. Still closer, the presence of the wall exerts geometrical restrictions on the kinematics of the fiber (excluded volume effect).

Fiber orientation close to a solid surface in a shear flow has been studied experimentally and the wall effect on fiber orientation has been documented in $[1,3,6,7]$. A rich variety of fiber behaviour has been observed. Typical example is the "pole vaulting" orbits during which the center of mass of the fiber is lifted from the wall. Experiments performed in shear flow down an inclined 
plane of a sedimenting fiber suspension suggest that short fibers (fiber aspect ratio $r_{a} \approx 10$ ) align with the vorticity axis. Pole vaulting is only observed if the solid wall is ridged $[1,3]$.

While several studies have been devoted to fiber level simulations, so far no Jeffery like wall effect model is available [2]. The need for such models is obvious, see [11] where a phenomenological recoiling model is proposed. The slow-down of Jeffery's orbits has been considered phenomenologically in the reduced strain closure model, cf. [10]. However, that model is developed in a different context (highly concentrated suspensions away from the wall).

This paper is organized as follows. Sections 2 and 3 are devoted to reviewing Jeffery's equation and the mesoscale framework for a fiber ensemble. The next section contains the bulk of this work where we propose modifications to Jeffery's equation to include the excluded volume effect. Scenarios of fiber sticking to the wall and recoiling from the wall are considered. The corresponding mesoscale (Fokker-Planck) equations are formulated in Section 5. We close the article with some numerical examples and conclusions.

\section{Microscale Framework}

In the original paper (see [4]) George Barker Jeffery considers the dynamics of a single prolate ellipsoid shaped particle immersed in a Newtonian liquid. The free flow velocity $v$ satisfies the incompressible Stokes equation. Let $\kappa=\nabla v$ so that the rate of deformation tensor is $\dot{\gamma}=\kappa+\kappa^{T}$ and the vorticity tensor is $\omega=\kappa-\kappa^{T}$. The center of mass of the particle is translated with velocity $v$ and the rate of rotation is given by Jeffery's equation:

$$
\dot{p}=-\frac{1}{2} \omega p+\frac{1}{2} \lambda[\dot{\gamma} p-(p \otimes p)(\dot{\gamma} p)]
$$

Here $p$ is the unit fiber orientation vector, $\lambda=\frac{r_{a}^{2}-1}{r_{a}^{2}+1}$ is a fiber geometry parameter dependent on the aspect ratio $r_{a}$ of the fiber length $\ell$ to the diameter. One can rewrite Jeffery's equation in a compact form [9]:

$$
\dot{p}=(I-p \otimes p) \cdot(M p) \equiv J(p),
$$

where the effective velocity gradient is defined as

$$
M=\frac{\lambda+1}{2} \kappa+\frac{\lambda-1}{2} \kappa^{T} .
$$

Jeffery's equation is commonly used as a basic ingredient for statistical mechanics models for fiber-fluid interaction. Such models generally inherit unphysical behaviour near obstacles from Jeffery's equation, see Fig. 1.

\section{Mesoscale Framework}

We give a brief introduction to the continuum description of fiber suspensions. The macroscopic velocity $v(x, t), x \in \Omega \subset \mathbb{R}^{3}, t>0$, of the suspension is governed by the equations $\nabla \cdot v=0, \rho v_{t}+\rho v \cdot \nabla v=\mu \Delta v-\nabla p+f+\nabla \cdot \sigma$ where 


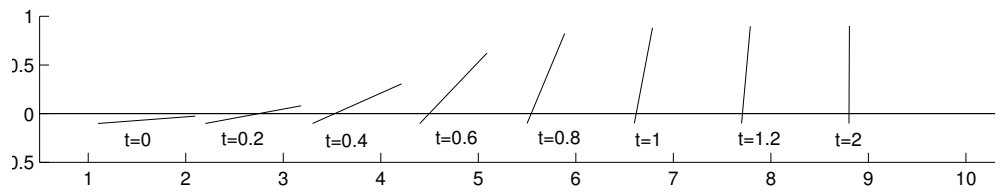

Figure 1. Unphysical situation: fiber penetrates the wall $y=0$ according to Jeffery's model.

the stress stored in the fibers $\sigma$ is a function of the rate of deformation tensor $\dot{\gamma}$ and the local fiber orientation distribution $\psi=\psi(p, x, t)$, with the particle number $N_{p}$ :

$$
\sigma=N_{p} \dot{\gamma}:\left\langle p^{4}\right\rangle ; \quad\left\langle p^{4}\right\rangle_{i j k l}(x, t)=\int_{S^{2}} p_{i} p_{j} p_{k} p_{l} \psi(p, x, t) d p .
$$

The evolution of $\psi(p, x, t), p \in S^{2}, x \in \Omega, t>0$ is governed by a FokkerPlanck equation describing Jeffery's dynamics $\dot{p}=J(p)$ superposed by isotropic (e.g. Folgar-Tucker model with scalar constant $C_{D}$ ) or anisotropic [8] orientation diffusion:

$$
\psi_{t}+v \cdot \nabla_{x} \psi=-\nabla_{p} \cdot(J(p) \psi)+\frac{1}{2} \nabla_{p} \cdot\left(C_{D} \nabla_{p} \psi\right)
$$

supplemented with an initial condition and appropriate boundary conditions on the inflow boundary. No boundary conditions wrt. $p$ are required since $p$ is defined on the unit sphere. The order of the model can be reduced by expanding $\psi$ in the components of even order moments $\left\langle p^{2 k}\right\rangle$ and obtaining a system of ODEs on the streamlines; this system requires a closure approximation.

\section{Single Fiber Dynamics: Modified Jeffery's Equations}

We now turn to modeling the collision of a fiber with a flat wall. In general, the collision influences both the translational and rotational motion of the fiber. However, we first proceed to describe pure rotational response and pure translation response.

\subsection{Kinematics of the fiber}

Our main assumptions are as follows:

1. The fibers are rigid i.e., inflexible and do not break. The wall is assumed to be flat. Thus the coordinates of the fiber with respect to the wall can be reduced to $(h, p)$ where $h$ denotes the distance from the center of mass of the fiber to the wall and $p$ is the orientation vector.

2. If the fiber is not in contact with the wall then Jeffery's equation (2.1) holds and $\dot{h}=\langle v, n\rangle \equiv \dot{h}_{J}$, where $v$ is the bulk velocity of the suspension flow, see [5], and $n$ is the inwards pointing normal vector of the wall. 
3. Since we mainly deal with slender fibers, the diameter of the fiber is neglected in comparison with the fiber length $\ell$, thus the contact condition is established by the centerline of the fiber touching the wall.

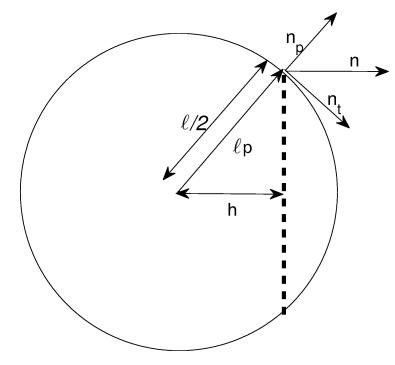

Figure 2. Contact condition. The fiber with orientation $p$ is in contact with wall (dashed line). Wall normal $n$ is decomposed in tangential and normal components to the sphere.

The Jeffery's equation treats fiber orientation $p$ and $-p$ equally; let us assume for the rest of this section that $p$ is chosen so that $\langle p, n\rangle \geq 0$ (see Fig. 2). Three cases are possible:

$$
\begin{aligned}
& |\langle p, n\rangle|<2 h / \ell \quad \text { no contact (allowed region), } \\
& |\langle p, n\rangle|=2 h / \ell \quad \text { contact with the wall (condition } \mathrm{C}) \\
& |\langle p, n\rangle|>2 h / \ell \quad \text { penetration (forbidden region) }
\end{aligned}
$$

where $\langle\cdot, \cdot\rangle$ denotes dot product in Euclidean space $\mathbb{R}^{3}$.

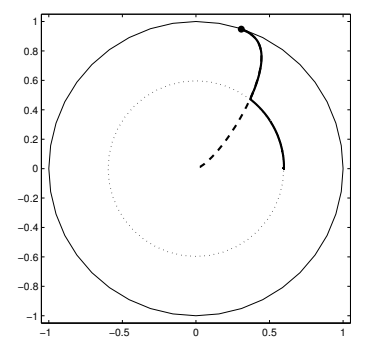

Figure 3. Trajectory $p(t)$ : Jeffery's equation (dashed line), sliding model (continuous line). Projection of sphere onto $O_{x y}$. Wall's normal is in $z$ direction.

We will model several types of fiber orbit near a wall:

1. Sliding (collision only influences $p$, see Fig. 3);

2. Pole vaulting (collision only influences $h$, see Fig. 4); 


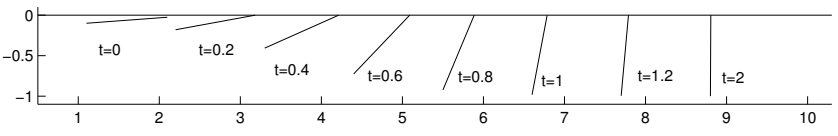

Figure 4. Pole vaulting model. Position of fiber wrt. wall in different time moments.

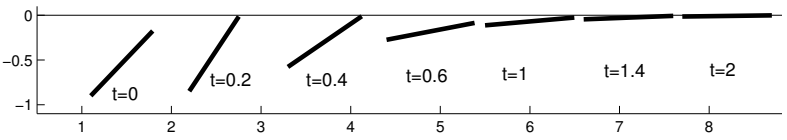

(a)

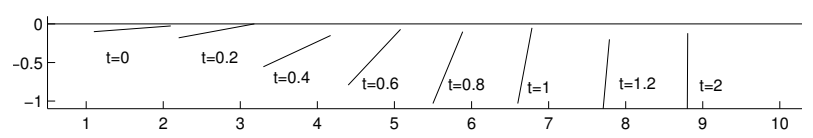

(b)

Figure 5. Fiber drifting $(\dot{h}<0)$ towards the wall $(y=0)$ and remaining in contact (a) and recoiling from the wall (b).

3. Combination of sliding and pole vaulting (both $p$ and $h$ are influenced, fiber remains in contact with wall after collision, see Fig. 5a);

4. Recoiling (fiber loses contact with wall right after collision, see Fig. 5b).

\subsection{First model: sliding}

We assume that during contact one end of the fiber is sliding along the wall as in Fig. 3. Jeffery's equation has to be modified as to cancel the advance of the end of the fiber towards the wall due to the translational and rotational motion of the fiber. The relative velocity of the nearest end of the fiber wrt. the wall is $\dot{\chi}:=\dot{h}+\frac{\ell}{2}\langle J(p), n\rangle$, thus the contact condition $\mathcal{C}$ is $|\langle p, n\rangle|=\frac{2 h}{\ell}$ and $\dot{\chi}<0$.

We take tangent vector $n_{t} \in T S_{p}^{2}$ such that $n_{t} \perp p,\left\langle n_{t}, n\right\rangle>0$ and $n \perp$ $n_{t} \times p$, see Fig. 2. With $n_{p}=\langle p, n\rangle p$ it is expressed:

$$
n_{t}=\frac{n-n_{p}}{\left\|n-n_{p}\right\|}=\frac{n-\langle n, p\rangle p}{\|n-\langle n, p\rangle p\|},
$$

where $\|\cdot\|$ is usual Euclidean norm. The modified Jeffery's equation is now constructed from two terms. Assuming $\dot{h}=0$ we see that the term needed to avoid penetration is $-\langle n, J(p)\rangle n_{t}$. Assuming $J(p)=0, \dot{h}<0$, the required term is $-\beta \dot{h}$, where

$$
\beta=\frac{\|n-\langle n, p\rangle p\|}{1-\langle n, p\rangle^{2}} .
$$


In the general case we write $\dot{p}=J(p)-(\langle n, J(p)\rangle+\beta \dot{h}) n_{t}$ under contact condition $\mathcal{C}$.

In total, the sliding model reads as:

$$
\dot{p}= \begin{cases}J(p) \equiv(I-p \otimes p) \cdot(M p), & \text { if } \neg \mathcal{C} ; \\ J(p)-(\langle n, J(p)\rangle+\beta \dot{h}) \frac{n-\langle p, n\rangle p}{\|n-\langle p, n\rangle p\|}, & \text { if } \mathcal{C} .\end{cases}
$$

Since $\left\langle p, \dot{p}_{J}\right\rangle=0$ and $\left\langle p, n_{t}\right\rangle=0$ from the construction of $n_{t}$, we conclude that $\langle p, \dot{p}\rangle=0$ holds for (4.5), thus the unit sphere is an invariant manifold.

\subsection{Second model: pole vaulting}

Here we assume that during contact $p(t)$ still fulfills Jeffery's equation and modify the equation for $h$ to avoid unphysical situations (see Fig. 1). geometrical constraint for single fiber. However, recoiling effect does not appear, i.e. the end of fiber remains on the wall. The center of mass of a fiber in a pole vault orbit $h(t)$ is no longer driven by the free flow velocity $v$, but by the contact itself. The equation follows from the relative velocity of the fiber end wrt. wall and reads as

$$
\dot{h}= \begin{cases}\dot{h}_{J} \equiv\langle v, n\rangle & \text { if } \neg \mathcal{C} ; \\ \frac{\ell}{2}|\langle\dot{p}, n\rangle| & \text { otherwise. }\end{cases}
$$

\subsection{Combination of sliding and pole vaulting}

The most general rigid body dynamics is a combination of translation and rotation. We obtain a class of models depending on a constant $\alpha \in[0 ; 1]$ interpolating between the two models introduced above:

$$
\dot{p}= \begin{cases}J(p) \equiv(I-p \otimes p) \cdot(M p), & \text { if } \neg \mathcal{C} ; \\ J(p)-\alpha(\langle n, J(p)\rangle+\beta \dot{h}) \frac{n-\langle p, n\rangle p}{\|n-\langle p, n\rangle p\|}, & \text { if } \mathcal{C}\end{cases}
$$

along with (4.6).

\subsection{Third model: recoiling}

We assume that fiber can slightly recoil from the wall at the moment of contact due to the impact and possible roughness of the wall, see Fig. 5. A fiber in Jeffery's theory is inertialess, thus impulsive equations are required. Let us consider 2 variants.

R1. The function $h$ jumps at the moment of contact, evolution of $p$ changes smoothly according to Jeffery's equation, see Fig. 5

$$
\begin{array}{ll}
\dot{h}=\langle v, n\rangle, & \text { if }|\langle p(t), n\rangle|<2 h(t) / \ell ; \\
h(t+0)=h(t)+c|\langle J(p(t)), n\rangle|, & \text { if }|\langle p(t), n\rangle|=2 h(t) / \ell,
\end{array}
$$

where $c \in \mathbf{R}^{+}$is a parameter. 
R2. Both functions $h$ and $p$ jump at the moment of a contact.

$$
\begin{aligned}
& \dot{p}=(I-p \otimes p) \cdot(M p), \quad|\langle p(t), n\rangle|<2 h(t) / \ell ; \\
& p(t+0)=\mathcal{P}(t), \quad|\langle p(t), n\rangle|=2 h(t) / \ell ; \\
& \dot{h}=\langle v, n\rangle \quad \text { if }|\langle p(t), n\rangle|<2 h(t) / \ell ; \\
& h(t+0)=\mathcal{H}(t), \quad \text { if }|\langle p(t), n\rangle|=2 h(t) / \ell,
\end{aligned}
$$

where $\mathcal{P}$ and $\mathcal{H}$ are functions such that $|\langle\mathcal{P}, n\rangle| \leq 2 \mathcal{H} / \ell$.

The pole vault model (4.6) is a special case of Model R1 with continuous solutions. The model R2 is much more general - both $p$ and $h$ can jump in case of contact.

The functions $\mathcal{P}, \mathcal{H}$ in $\mathrm{R} 1, \mathrm{R} 2$ have to be provided according to the situation. These can be quite sophisticated and include fiber diffusion towards the wall so that $h$ decreases with a jump and $p$ is adjusted. An example of $\mathcal{P}, \mathcal{H}$ is provided in Section 6.

\section{$5 \quad$ Mesoscale Modeling}

Let us consider a stationary filtration problem where a fiber suspension flows through a semi-permeable membrane and the fibers cannot flow through the membrane. For the simplicity of argument assume that each streamline of macroscopic velocity can be parameterized by distance from the membrane $h \in[0, H]$. We derive a Fokker-Planck equation on each streamline wrt. the distribution $\psi(p, h, t), p \in S^{2}, h \in(0, H), t>0$.

For each value of $h$ we partition the unit sphere according to (4.1)-(4.3):

$$
A(h)=\left\{p \in S^{2}||\langle p, n\rangle \mid<2 h / \ell\right\}, \quad C(h)=\partial A, \quad E(h)=S^{2} \backslash \bar{A} .
$$

The support of $\psi(\cdot, h, \cdot)$ is contained in the closure of $A(h)$.

Choose one of the continuous microscale models (4.5)-(4.7). The FokkerPlanck equation reads as:

$$
\psi_{t}=-\nabla_{p} \cdot(\dot{p} \psi)-\nabla_{h}(\dot{h} \psi)+\frac{1}{2} \nabla_{p} \cdot\left(C_{D p} \nabla_{p} \psi\right)+\frac{1}{2} \nabla_{h} \cdot\left(C_{D h} \nabla_{h} \psi\right),
$$

where $C_{D p}$ is the Fokker-Planck orientation diffusion constant and $C_{D h}$ is the diffusion constant for the centroids of the fiber. The equation requires an initial condition and boundary conditions on the inflow boundaries.

If the diffusion constants $C_{D p}$ and $C_{D h}$ vanish, no boundary conditions either wrt. $p$ or $h$ are required near the wall since the microscale equations allow for no flux. The fiber ends may remain in contact with the wall for prolonged time periods, therefore the edge of zero surface $C \subset S^{2}$ may contain a finite mass. For this reason, $\psi$ must be interpreted as a distribution.

For nonzero diffusion constants homogeneous Neumann (no flux) condition has to be specified for $(p, h) \in S^{2} \times[0, h]$ s.t. contact condition $\mathcal{C}$ holds. 


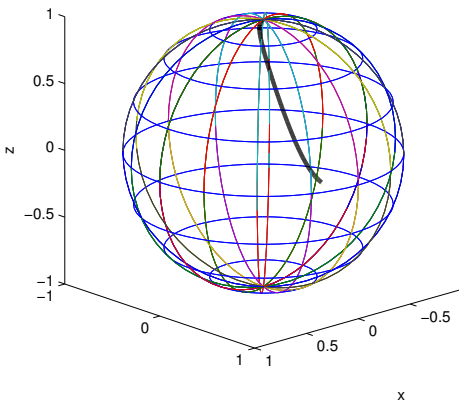

Figure 6. Trajectory of orientation vector, Jeffery's equation.

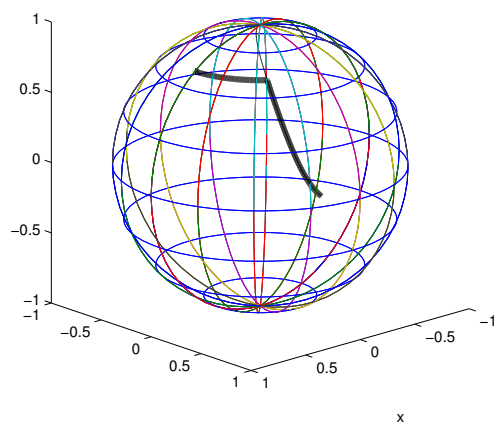

Figure 8. Trajectory of orientation vector, sliding model.

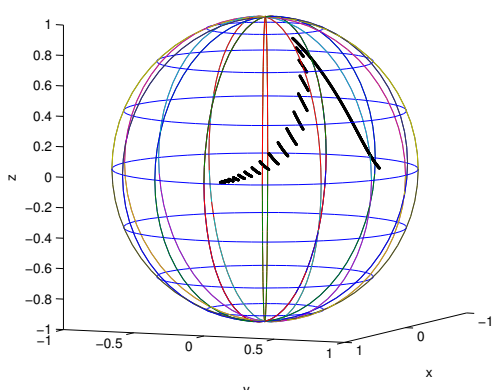

Figure 7. Fiber orientation, with recoiling model $\mathrm{R} 2$.

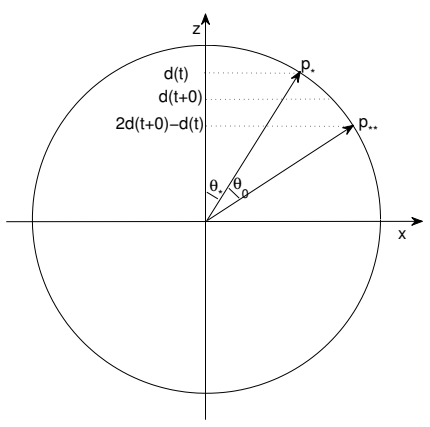

Figure 9. In recoiling models the orientation jumps from $p_{*}$ to $p_{* *}$.

\section{Examples}

We give some numerical examples. We choose matrix $M$ in (2.1) as

$$
M=\left(\begin{array}{lll}
-1 & 0 & 0 \\
0 & -3 & 0 \\
0 & 0 & 4
\end{array}\right)
$$

There are two stable equilibrium points for Jeffery's equation $(0 ; 0 ; \pm 1)$. We take the wall's normal as north pole $n=(0 ; 0 ; 1)$. For simplicity, we assume that $\dot{h}=0$, i.e., simple shear flow is given.

We have used fourth order Runge-Kutta method for numerically solving the ODEs. The contact condition is checked for each time step. Examples for dynamics of the orientation vector $p$ are shown in Figs. 6, 8, 7. Fiber dynamics against plane $O_{x y}$ are plotted in Figs. 5, 4. In Fig. 6, both pole vaulting (4.6) and recoiling model (4.8) as well as the single fiber orientation 
without wall influence are represented. However, we see difference between the two models when comparing evolution of $h$, where for one case fiber stays in contact with the wall (Fig. 4), and for second case fiber slightly recoil from the wall (Fig. 5b).

In Fig. 5, an example of a situation with recoiling, described with equations (4.10) and (4.9), is given. Let us specify the terms $\mathcal{P}, \mathcal{H}$. We first describe the situation when the wall normal points in the $z$ direction, where the recoiling is described by a jump of orientation from $p_{*}$ to $p_{* *}$, see Fig. 9. Next we rotate the coordinates so that $(0,0,1)$ is mapped to the actual $n$. We use spherical coordinates on the unit sphere: $x=\sin (\theta) \sin (\phi), y=\sin (\theta) \cos (\phi), z=\cos (\theta)$. Let $(\phi, \theta)$ and $\left(\phi_{n}, \theta_{n}\right)$ represent the vectors $p$ and $n$ respectively. We choose $\mathcal{H}=\max \left\{h(t)-c\left|\left\langle\dot{p}_{J}, n\right\rangle\right|, 0\right\}$. We will inspect the case when $p_{z}(t)>0$ noting that derivation is analogous if $p_{z}<0$. To compute $\mathcal{H}$, the first step is to rotate $n$ to $(0 ; 0 ; 1)$. To achieve the aim, we rotate $n$ by an angle $-\phi_{n}$ around $z$ (rotation matrix $\left.R_{z,-\phi_{n}}\right)$ and then by an angle $-\theta_{n}$ around $y$. We obtain $(0 ; 0 ; 1)=$ $R_{y,-\theta_{n}} R_{z,-\phi_{n}} n$. Similarly, we transform $p(t)$ into $p_{*}$ as $p_{*}=R_{y,-\theta_{n}} R_{z,-\phi_{n}} p(t)$. Then we rotate $p_{*}$ by an angle $\theta_{0}$ against a plane $O_{x y}$, see Fig. 9. Denote $\left(\phi_{*}, \theta_{*}\right)$ - vector $p_{*}$ in a polar coordinate system. We assume that $2 h(t+0)-$ $h(t)=\cos \left(\theta+\theta_{0}\right)$, see Fig. 9. Hence we get $\theta_{0}=\arccos (2 h(t+0)-h(t))-\theta_{*}$. The case $2 h(t+0)-h(t) \leq 0$ is a special case, in which we take $\theta_{0}=\pi / 2-\theta_{n}$. Finally the vector $p_{*}$ rotated by an angle $\theta_{0}$ around the plane $O_{x y}$ reads as

$$
p_{* *}= \begin{cases}R_{z, \phi_{*}} R_{y, \arccos (2 h(t+0)-h(t))-\theta_{*}} R_{z,-\phi_{*}} p_{*}, & 2 h(t+0)-h(t)>0 ; \\ R_{z, \phi_{*}} R_{y, \pi / 2-\theta_{n}} R_{z,-\phi_{*}} p_{*}, & 2 h(t+0)-h(t) \leq 0 .\end{cases}
$$

Finally we transform $p_{* *}$ from (6.2) back to the $p(t+0)$ as

$$
p(t+0)=R_{z,-\phi_{n}}^{-1} R_{y,-\theta_{n}}^{-1} p_{* *} .
$$

In that way we have obtained $\mathcal{P}, \mathcal{H}$ for (4.9), (4.10) as follows:

$$
\begin{gathered}
\mathcal{P}=R_{z,-\phi_{n}}^{-1} R_{y,-\theta_{n}}^{-1} R_{z, \phi_{*}} R_{y, \arccos (h(t)-2 h(t+0))-\theta_{*}} R_{z,-\phi_{*}} R_{y,-\theta_{n}} R_{-\phi_{n}} p(t), \\
\mathcal{H}=\max \left\{h(t)-c\left|\left\langle\dot{p}_{J}, n\right\rangle\right|, 0\right\},
\end{gathered}
$$

where $(\phi, \theta),\left(\phi_{n}, \theta_{n}\right)$ and $\left(\phi_{*}, \theta_{*}\right)$ describe vector $p, n$ and $R_{x, \theta} R_{z,-\phi} p(t)$, correspondingly, in a polar coordinate system.

Fig. 4a shows the real positions of a fiber at different time moments that we obtained using the recoiling model. The wall is assumed to be semi-permeable so that $h$ decreases with time. The trajectory traced out by $p(t)$ is shown in Fig. 10.

\section{Conclusions}

This study has shown that Jeffery's equation can be modified so that the fibers never penetrate a rigid wall. We believe that incorporating the distance from the wall $h$ in the description of suspensions is important and can be extended e.g., to filtration where a membrane plays the role of the wall, see Fig. 5a. 
The complexity of the involved processes has not allowed us to formulate a single model, - the choice between a model with or without recoiling will depend among other factors on the physical characteristics (roughness) of the wall, flexibility of the fibers, viscosity of the liquid. Of course, a direct two phase simulation of interaction between the fibers and the liquid could provide much more exact results, however, the value of our models is in the simplicity and numerical tractability in applications.

The mesoscale formulation is readily obtained in the form of a FokkerPlanck equation. However, the numerical treatment of it in the style of FolgarTucker models (where low order moment approximations are used) is more challenging. However, one can modify any Folgar-Tucker equation directly as we did here with Jeffery's equation.

We have not touched the problem of estimating the parameter $\alpha$ in (4.7) from experimental data.

\section{References}

[1] A. Carlsson, F. Lundell and D. Soderberg. The wall effect on the orientation of fibres in a shear flow. Ann. Trans. Nordic Rheol. Soc., 14:1-7, 2006.

[2] S. Dong, X. Feng, M. Salcudean and I. Gartshore. Concentration of pulp fibers in 3D turbulent channel flow. Int. J. Multiphase Flow, 29:1-21, 2003. http://dx.doi.org/10.1016/S0301-9322(02)00128-3.

[3] R. Holm and D. Soderberg. Shear influence on fibre orientation. Rheol. Acta, 46:721-729, 2007. http://dx.doi.org/10.1007/s00397-007-0166-y.

[4] G.B. Jeffery. The motion of ellipsoidal particles immersed in a viscous fluid. Proc. R. Soc. Lond. Ser. A, 102:161-179, 1922.

[5] M. Junk and R. Illner. A new derivation of jeffery's equation. J. Math. Fluid Mech., 9:455-488, 2005. http://dx.doi.org/10.1007/s00021-005-0208-0.

[6] X.K. Ku and J.Z. Lin. Effect of two bounding walls on the rotational motion of a fiber in the simple shear flow. Fibers and Polymers, 10:302-309, 2009. http://dx.doi.org/10.1007/s12221-009-0302-7.

[7] X.K. Ku and J.Z. Lin. Inertial effects on the rotational motion of a fibre in simple shear flow between two bounding walls. Phys. Scr., 80:025801 (10 pp), 2009.

[8] A. Latz, U. Strautins and D. Niedziela. Comparative numerical study of two concetrated fiber suspension models. J. Non-Newtonian Fluid Mech., 165:764781, 2010. http://dx.doi.org/10.1016/j.jnnfm.2010.04.001.

[9] J. Linn. On the frame-invariant description of the phase space of folgar-tucker equation. Progress in Industrial Mathematics at ECMII 2002, pp. 327-332, 2004 .

[10] J.H. Phelps and C.L. Tucker. An anisotropic rotary diffusion model for fiber orientation in short - and long fiber thermoplastics. J. Non-Newtonian Fluid Mech., 156:165-176, 2009. http://dx.doi.org/10.1016/j.jnnfm.2008.08.002.

[11] S.L. Zhang, J.Z. Lin and W.F. Zhang. Numerical research on the fiber suspensions in a turbulent t-shaped branching channel flow. Chin. J. Chem. Eng., 15:30-38, 2007. http://dx.doi.org/10.1016/S1004-9541(07)60030-5. 